\title{
Conceptual framework for development of a toolkit for prevention of work-related musculoskeletal disorders
}

\author{
Wendy Macdonald* \\ Centre for Ergonomics \& Human Factors, Faculty of Health Sciences, La Trobe University, Victoria 3086, \\ Australia
}

\begin{abstract}
The World Health Organisation (WHO) network of Collaborating Centres in Occupational Health aims to promote the development, implementation use of "toolkits" for workplace use in reducing the risk of injuries and disease. As a major partner within this network, the International Ergonomics Association is committed to developing a toolkit to reduce the risk of musculoskeletal disorders. This paper outlines the kind of conceptual framework required to support this work.
\end{abstract}

Keywords: Musculoskeletal disorders, causal models, physical hazards, psychosocial hazards, risk management, intervention effectiveness.

\section{Introduction}

The World Health Organisation (WHO) network of Collaborating Centres in Occupational Health aims to promote the development, implementation use of "toolkits" for workplace use in reducing the risk of injuries and disease. As a major partner within this network, the International Ergonomics Association is committed to developing a toolkit to reduce the risk of musculoskeletal disorders.

According to this WHO network, such a toolkit might focus on a particular kind of hazard, or on a particular kind of risk such as that of musculoskeletal disorders in the present case. The toolkit should provide practical tools and strategies for workplace use in identifying hazards and assessing risk, and for developing, implementing and evaluating interventions to reduce risk. Where appropriate this should include training materials and guidance documents to support effective implementation of the risk management process.

Development of such a toolkit to help prevent musculoskeletal disorders is a major undertaking, because research evidence is now very clear that the risk of such disorders or injuries can stem from a large and diverse set of hazards - including but certainly not confined to the physical hazards associated with 'manual handling' activities. Further, it has been shown that interactions between hazards of all kinds can substantially affect MSD risk [1-3]. This means that the extent of a particular hazard exposure, if considered independently of other hazard exposures and risk factors, is not necessarily a good indicator of overall MSD risk.

\section{Models of MSD Causation}

The characteristics of MSD risk outlined above make it essential for the planned toolkit to utilise an holistic, evidence-based conceptual framework so that all relevant sources of risk are given due consideration. There are many examples of such frameworks. For example, the model in Figure 1 was based on a review of research evidence by a multidisciplinary expert committee on behalf of the USA National Research Council and Institute of Medicine [4].

E-mail: w.macdonald@1atrobe.edu.au. 


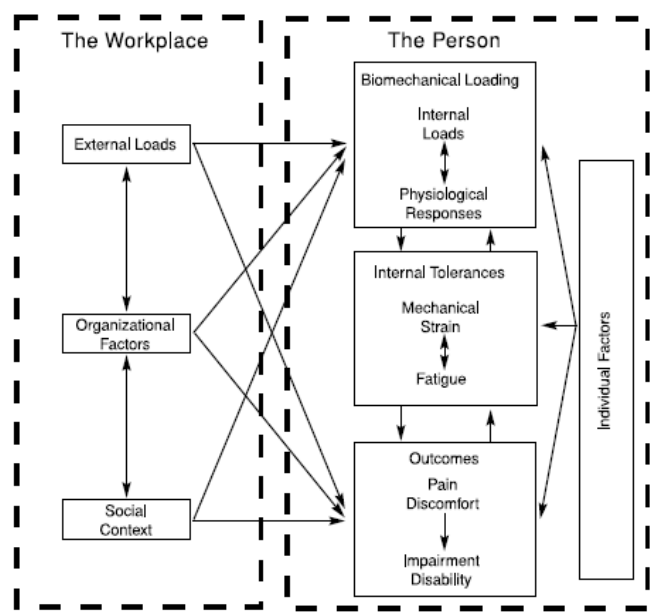

Figure 1. Model of MSD hazards and risk factors developed by US National Research Council [reproduced from 4]

Figure 1 depicts three groups of workplace hazards: external (biomechanical) loads, organizational factors, and social context; those within the latter two groups are commonly known as psychosocial hazards. Hazards within all three categories interact with each other (shown by linking arrows) and all of these hazards can affect processes internal to individual workers (internal biomechanical loading, physiological responses) and personal outcomes (discomfort, pain, impairment, disability). As shown on the right of the diagram, individual factors influence all personal processes and outcomes.

It can also be seen in The Person section of Figure 1 that 'Fatigue' is recognized as a relevant factor. 'Stress' is not highlighted here, although it is implicit within 'Physiological Responses'. The well documented role of stress in MSD causation is illustrated more clearly in Figure 2, which highlights the interacting effects on MSD risk of 'physical' hazards (mainly biomechanical) and psychosocial hazards. A person's internal 'stress response', as shown here, occurs when situations are experienced as stressful; it is multidimensional, with physiological and behavioural, as well as cognitive and affective dimensions [5], with potentially profound effects on health, including MSD risk [e.g. 6-8].

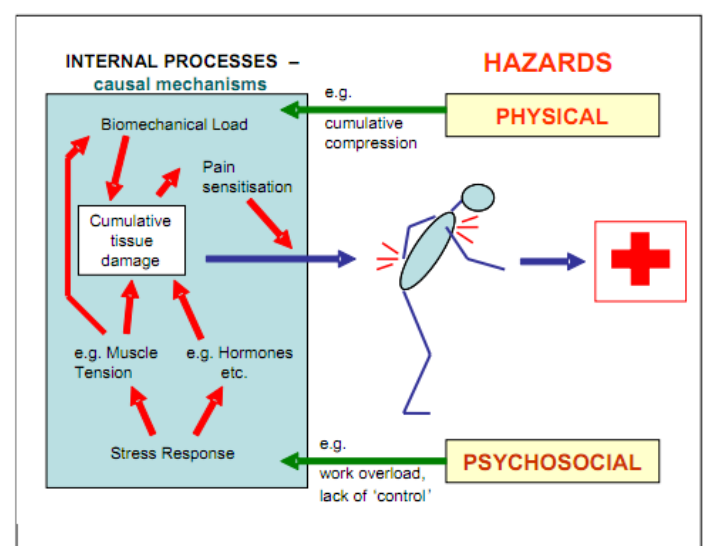

Figure 2. A model highlighting evidence that internal processes producing cumulative tissue damage include stress. [Reproduced from 8, p.9]

The primary purpose of the models in Figures 1 and 2 is to promote better understanding of MSD aetiology, based on current research evidence.

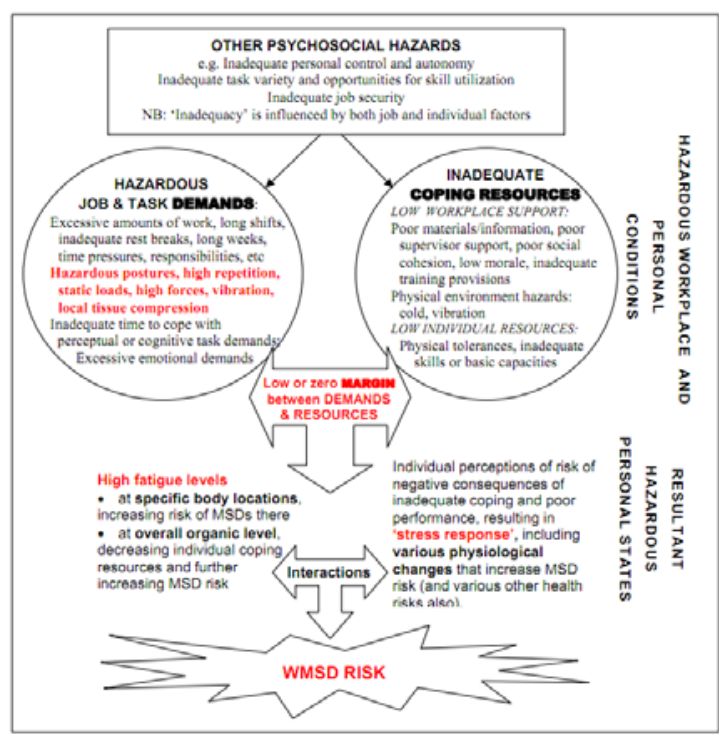

Figure 3. A composite model, modified from [8, Figure 6].

The model in Figure 3 is in accord with these but is more directly applicable to workplace risk management because it provides more specific detail concerning the wide range of work-related hazards that can combine to affect risk. This model shows that MSD risk is increased if Job and Task Demands are hazardous or excessive in relation to available Coping Resources, and that risk is also affected by Other Psychosocial Hazards. The physical hazards of manual task performance are included within $J o b$ and Task Demands, along with the cognitive and emo- 
tional demands of task performance, and the broader demands of the overall job. Coping Resources are determined both by workplace factors (support systems and resources; psychosocial and physical environment influences) and by the individual's own capabilities. Importantly, it is the combination of these diverse variables that determines risk.

\section{Implications for toolkit content}

The above models make clear that the proposed toolkit needs to encompass tools for the identification and assessment of a very wide range of variables. Potentially important factors relate to work organization, job design and the workplace environment (psychosocial as well as physical), and workers' levels of fatigue and stress also need consideration, since internal physiological and biomechanical dimensions of these are linked to MSD risk.

Despite such evidence, recent research in workplaces of four large Australian companies in high risk industry sectors found there was little attention to psychosocial hazards [9]. The focus was on manual handling, and in two of the four there was particular emphasis on training in 'safe' movement techniques despite strong research evidence that this typically does not reduce MSD risk. Published research concerning practices of consultant ergonomists in the UK indicates a similar situation there [10]. This situation is promoted by Manual Handling Standards, Codes of Practice and Associated Guidance Materials, which typically provide very little coverage of psychosocial hazards.

Further, the particular combination of hazards present in a given situation needs to be taken into account. The importance of this kind of approach is further substantiated by research evaluating the effectiveness of various kinds of interventions intended to reduce MSD risk. For example, a 2008 review of such evidence concluded that:

... a combination of several kinds of interventions (multidisciplinary approach) including organisational, technical and personal/individual measures is better than single measures ... [and that] ... a participative approach which includes the workers in the process of change has a positive effect on the success of an intervention.

$[9, \mathrm{pp} .7-8]$

As well as hazard identification and risk assessment, the toolkit needs to support the process of selecting, implementing and evaluating the effectiveness of interventions.
These aspects of MSD risk management are particularly important in light of strong research evidence that many MSD interventions fail to result in the anticipated reductions in risk. Wells (12) has identified some of the research questions in need of investigation if we are to develop more effective interventions. The process of implementing, evaluating and further developing this toolkit might provide a good context for the IEA to support such progress.

Finally, in an earlier paper in this session, David Caple identified some of the more specific issues that will also influence toolkit content. These include the probable need for tools customised to suit the needs of different types of work, industry sectors, legislative and enforcement systems, and socio-economic contexts in both industrially developing and developed countries.

All of this presents many challenges to the IEA MSDs Technical Committee, which will need to establish processes to coordinate and where possible to provide support for this important, ongoing work.

\section{References}

[1] Bernard, B.P, (ed). (1997). Musculoskeletal Disorders (MSDs) and Workplace Factors. Cincinnati: National Institute for Occupational Safety and Health.

[2] National Research Council and Institute of Medicine (2001). Musculoskeletal disorders and the workplace: low back pain and upper extremities. Section on Methodological Issues and Approaches, pp.65-81. USA: National Academy Press. Retrieved May 2011 from http://www.nap.edu/openbook.php?record id=10032\&page $=$ $\underline{\mathrm{R} 2}$

[3] Marras, W.S. (2008). The Working Back: A Systems View. NJ: Wiley.

[4] USA National Research Council and Institute of Medicine

[5] Cox, T. (1978). Stress. London : Macmillan.

[6] Warren, N. (2001). Work stress and musculoskeletal disorder etiology: The relative roles of psychosocial and physical risk factors. Work, 17, 221-234.

[7] Aptel, M.,and Cnockaert, J. (2002) Stress and work-related musculoskeletal disorders of upper extremities. Trade Union Technical Bureau (TUTB) Newsletter 2002, no 19-20. Retrieved May 2011 from http://hesa.etuirehs.org/uk/newsletter/files/Newsletter-20.pdf

[8] Macdonald, W. and Evans, O. (2006). Research on the Prevention of Work-related Musculoskeletal Disorders. Stage 1 - Literature Review. Australian Safety \& Compensation Council, Canberra: Commonwealth of Australia. Retrieved May 2011 from http://www.safeworkaustralia.gov.au/ABOUTSAFEWORK AUSTRA-

LIA/WHATWEDO/PUBLICATIONS/Pages/RR200606Rese archOnPreventionOfWRMusculoskeletalDisorders.aspx

[9] Macdonald, W., Evans, O., and Armstrong, R. (2007). Research on the prevention of musculoskeletal disorders - stage 2. A study of a small sample of workplaces in high risk in- 
dustries. Department of Employment and Workplace Relations, Project No. CIR 1001439-07. Retrieved September 2011 from:

http://www.latrobe.edu.au/ergonomics/attachments/stage2report-ssos.pdf

[10] Whysall, Z. J., Haslam, R. A., and Haslam, C. Processes, barriers, and outcomes described by ergonomics consultants in preventing work-related musculoskeletal disorders. $A p$ plied Ergonomics, 2004, 35, 343-351.
[11] European Agency for Safety and Health at Work: Zinta Podniece (2008). Work-related Musculoskeletal Disorders: Prevention Report. Luxembourg: Office for Official Publications of the European Communities, EASHW. Retrieved May 2011 from

http://osha.europa.eu/en/publications/reports/en_TE8107132 ENC.pdf

[12] Wells, R. (2009). Why have we not solved the MSD problem? Work, 34, 117-121. 